\title{
Optimization of Surface Roughness in EDM for D2 Steel by RSM-GA Approach
}

\author{
J. Anitha ${ }^{1, *}$, Raja Das ${ }^{1}$, M. K. Pradhan ${ }^{2}$ \\ ${ }^{1}$ School of Advanced Sciences, VIT University, Vellore, Tamil Nadu-632014 \\ ${ }^{2}$ Maulana Azad National Institute of Technology, Bhopal, Madhya Pradesh-462051 \\ *Corresponding Author: anithasastry2077@gmail.com
}

Copyright $(\subseteq 2014$ Horizon Research Publishing All rights reserved.

\begin{abstract}
Modeling and optimization of machining parameters are very important in any machining processes. The current study provides predictive models for the functional relationship between various factors and responses of electrical discharge machined AISI D2 steel component. Surface Roughness (Ra) is important as it influences the quality and performance of the products, hence the minimization of surface roughness in manufacturing sectors is of maximum importance. It is also realistic and desirable if the finished parts do not need further any operations to meet the required optimum level of surface quality. For achieving the required optimum levels of surface quality, the proper selection of machining parameters in EDM is essential. Four significant machining parameters, Ip (Pulse Current), Ton (Pulse on Time), Toff (Off Time) and V (Gap Voltage) in the EDM process have been selected and with the various combination experiments were conducted. A mathematical regression model was developed to predict the average Surface Roughness in electrical discharge machined surface. The developed model was validated with new experimental data. The model was coupled with genetic algorithm to predict the minimum possible surface roughness. It is found that the predicted and experimental values were close to a certain extent, which specifies that the established model can be successfully used to predict the surface roughness. Also, the developed model could be used for the selection of the levels in the EDM process for saving in machining time and product cost can be achieved by utilizing the model.
\end{abstract}

Keywords Electrical Discharge Machine, Surface Roughness, Response Surface Methodology, Genetic Algorithms

\section{Introduction}

Electrical discharge machining grew over the last few decades from uniqueness to a mainstream manufacturing method. It is most widely and successfully applied to the machining of a varied piece of work material in the advance industry [1]. EDM has a high capability of machining the precise cavities of dies and moulds. However, the surface quality aspect of EDMed component is important to meet the requirements of component performance, longevity and reliability [2]. In order to increase the machining efficiency, the surface roughness must be minimized in the EDM process. Therefore, in EDM it is necessary to minimize the surface roughness by optimizing the process parameters. Usually, the necessary parameters are chosen either through handbooks or through experience and with respect to different machining parameters followed by parametric optimization of the process. Therefore, in EDM it is necessary to minimize the surface roughness by optimizing the process parameters. Usually, the necessary parameters are chosen either through handbooks or through experience and with respect to different machining parameters followed by parametric optimization of the process. But, this is a tough task, hence statistical methods are employed to solve this problem.

In the past few years, several EDM modelling and optimization tools relating the process variables and surface roughness have been established. Bhattachrya et al. [3] implemented Response Surface Methodology (RSM) to develop a mathematical model for correlating the process parameter with the responses. Zarepour et al [4] investigated the effect of machining parameters of the EDM process, including on-time, current, voltage, the engaging time between workpiece and electrode, and pre-EDM roughing on electrode wear were experimentally investigated. Kao et al. [5] optimized the EDM parameters, for EWR, MRR and surface roughness as the performance characteristics of the EDM machining of Ti6Al4V alloy. Sanchez et. al [6] solved an inversion model, based on the least squares theory, which involves establishing the values of the EDM input to ensure the simultaneous fulfilment of various responses along with and surface roughness. Chen and Mahdavian, [7] represented various graphs to elucidate the relationship between the performance output results of SR. Mandal et. al [8] modeled and optimize the EDM process using soft computing techniques. Artificial neural network with back propagation 
algorithm is used to model the process. A multi-objective optimization method, non-dominating sorting genetic algorithm-II is used to optimize the process. Pradhan et al. [9], [10] established quite a few surface models based on various RSM models taking the effects of a number of parameters under consideration. They later developed a regression model, it was noted that the model produces a lot of reliable surface roughness prediction for a given work under different process conditions. Majumder [11] used regression equations of the polynomial type were calculated to predict the Electrode Wear Rate (EWR) obtained using GA- based RSM for optimization. Tzeng, and Chen, [12], analyzed the material removal rate, electrode wear ratio and the work piece surface finish on process parameters during the manufacture of SKD 61 by EDM.

It can be seen that a few work has been done on the in the combination of GA-based techniques, however, this combination of a hybrid method including a back-propagation neural network (BPNN), a GA, and RSM have never been tried on AISI D2 steel, hence proposed in this study to determine optimal parameter settings of the EDM process. It was found that the algorithm of GA approach has better prediction and confirmation results than the RSM method. In this article a GA-based RSM has been proposed to minimize the $\mathrm{Ra}$ in EDM process. A mathematical model was established to predict the relationship amongst the process parameter of EDM and response Ra by using RSM. A GA has been combined with this model to optimize the process parameters. As a consequence, optimal parameters that are required under various operational conditions of EDM were acquired.

\section{Experimental Setup}

Experiments were conducted to check the effect and further optimization of various machining parameters on surface roughness. These experiments were conducted to analyze the effects the chosen work material for the analysis work is AISI D2 (DIN 1.2379) tool steel which has a growing range of applications in the field of manufacturing tools in mould industries. Experiments were conducted on Electronica Electraplus PS 50ZNC die sinking machine. An electrolytic pure copper with a diameter of $30 \mathrm{~mm}$ was used as a tool electrode (positive polarity) and work piece materials used were steel square plates of dimensions $15 \times 15$ $\mathrm{mm}^{2}$ and of thickness $4 \mathrm{~mm}$. Commercial grade EDM oil ( specific gravity $=0.763$, freezing point $=94^{\circ} \mathrm{C}$ ) was used as dielectric fluid. Lateral flushing with a pressure of 0.3 $\mathrm{kgf} / \mathrm{cm}^{2}$ was used. The test conditions are depicted in Table 1 .

Table 1. -Different variables used in the experiment and their levels

\begin{tabular}{|c|c|c|c|}
\hline \multirow{2}{*}{ Variable } & \multicolumn{3}{|c|}{ Levels } \\
\cline { 2 - 4 } & 1 & 2 & 3 \\
\hline Discharge current (Ip) in A & 5 & 10 & 15 \\
\hline Pulse on time (Ton) in $\mu \mathrm{s}$ & 50 & 75 & 100 \\
\hline Pulse off Time (Toff) in $\mu \mathrm{s}$ & 575 & 1490 & 2400 \\
\hline Discharge Voltage (V) in volt & 40 & 45 & 50 \\
\hline
\end{tabular}

\section{Regression Model}

The Response surface methodology approach is the procedure for determining the relationship between various process parameters with various machining criteria. In this work also RSM is used to determine the relationship between the input process parameters of EDM with surface roughness [13]. In order to study the effects of EDM parameters, on surface roughness second order polynomial response surface mathematical model developed as shown in the Equation (1).

$$
\begin{aligned}
R a= & a_{0}+a_{1}(\text { Ip })+a_{2}(\text { Ton })+a_{3}(\text { Toff })+a_{4}(V)+b_{1}(\text { Ip })^{2}+b_{2}(\text { Ton })^{2}+b_{3}(\text { Toff })^{2}+b_{4}(V)^{2} \\
& +c_{1}(\text { Ip })(\text { Ton })+c_{2}(\text { Ip })(\text { Toff })+c_{3}(\text { Ip })(V)+c_{4}(\text { Ton })(\text { Toff })+c_{5}(\text { Ton })(V)+c_{6}(\text { Toff })(V)
\end{aligned}
$$

The coefficient $a_{0}$ is the constant term; the coefficients $a_{1}, a_{2} a_{3}$ and $a_{4}$ are the linear terms; the coefficients $b_{1}, b_{2}$ $b_{3}$ and $b_{4}$ are the quadratic terms; the coefficients $c_{1}, c_{2} c_{3}, c_{4}, c_{5}$ and $c_{6}$ are the interaction terms. The coefficients of regression model were estimated from the experimental results and the model is developed.

\section{Genetic Algorithm}

Genetic algorithm was used to obtain the optimum machining parameters for output Surface Roughness by using the several combinations of the input parameters. The computational algorithm was implemented in MATLAB code. Genetic algorithms (GAs) mainly work on three types of operators: reproduction, crossover, and mutation. Reproduction is accomplished by copying the best individuals from one generation to the next. The best solution is monotonically improving from one generation to the next. The selected parents are submitted to the crossover operator to produce one or two children. The crossover is carried out with an assigned probability, which is generally rather high. If a number randomly sampled is inferior to the probability, the crossover is performed. The genetic mutation introduces diversity in the population by an 
occasional random replacement of the individuals. The mutation is performed based on an assigned probability. A random number is used to determine if a new individual will be produced to substitute the one generated by crossover. The mutation procedure consists of replacing one of the decision variable values of an individual, while keeping the remaining variables unchanged. The replaced variable is randomly chosen, and its new value is calculated by randomly sampling within its specific range. The standard genetic algorithm is expressed as given below.

BEGIN

INITIALIZE population with a random candidate solution

EVALUATE each candidate;

REPEAT UNTIL (terminate conditions) is satisfied DO

1. SELECT parents;

2. RECOMBINE pairs of parents;

3. MUTATE the resulting offspring;

4. SELECT individuals or the next generation;

END

\section{Optimization of EDM parameters}

In the present study, GA is used as an optimization technique for solving a bound-constrained optimization problem. The regression models developed by response surface methodology have been used as objective function and the upper and lower bound parameters are identified by conducting experiments. The problem can be formulated as given below.

Minimize

$$
\begin{aligned}
R a= & 5.4035+0.1997(\text { Ip })+0.0268(\text { Ton })+0.0002(\text { Toff })-0.0853(V)+0.0028(\text { Ip })^{2} \\
& -0.0005(V)^{2}+0.0006(\text { Ip })(\text { Ton })-0.0017(\text { Ip })(V)-0.0002(\text { Ton })(V)
\end{aligned}
$$

Subject to

$5 \leq$ Ip $\leq 15$

$50 \leq$ Ton $\leq 100$

$575 \leq$ Toff $\leq 2400$

$40 \leq V \leq 50$

Table.2 ANOVA summary for Surface Roughness

\begin{tabular}{|c|c|c|c|c|}
\hline Term & Coef & SE Coef & T & P \\
\hline Constant & 6.00359 & 0.005805 & 1034.272 & 0.000 \\
\hline Ip & 1.11382 & 0.003166 & 351.789 & 0.000 \\
\hline Ton & 0.59574 & 0.010348 & 57.569 & 0.000 \\
\hline Toff & 0.08128 & 0.011329 & 7.174 & 0.000 \\
\hline V & -0.37323 & 0.003166 & -117.883 & 0.000 \\
\hline Ip $\times$ Ip & 0.06614 & 0.007887 & 8.386 & 0.000 \\
\hline Ton $\times$ Ton & 0.01594 & 0.009362 & 1.703 & 0.119 \\
\hline Toff $\times$ Toff & -0.08097 & 0.028829 & -2.809 & 0.019 \\
\hline $\mathrm{V} \times \mathrm{V}$ & 0.00809 & 0.007887 & 1.026 & 0.329 \\
\hline$($ Ip $) \times($ Ton $)$ & 0.06762 & 0.004222 & 16.016 & 0.000 \\
\hline$($ Ip $) \times($ Toff & 0.00881 & 0.005783 & 1.524 & 0.158 \\
\hline$($ Ip $) \times(V)$ & -0.04224 & 0.003149 & -13.416 & 0.000 \\
\hline$($ Ton $) \times($ Toff $)$ & 0.04873 & 0.028849 & 1.689 & 0.122 \\
\hline$($ Ton $) \times(V)$ & -0.02180 & 0.004222 & -5.162 & 0.000 \\
\hline$($ Toff $\times(V)$ & -0.00239 & 0.005783 & -0.413 & 0.688 \\
\hline
\end{tabular}




\section{Result and Discussion}

ANOVA is used to check the sufficiency of the second order model, which includes test for the significance of the regression model and model coefficients. It is used for testing the null hypothesis of the experimental data with a confidence level of $95 \%$. The $p$-value for the $F$-statistic, expressing the probability of observing a value of $F$ at least, as large, if $H_{0}$ is true and treatments have no effect. If $p$-value less than 0.05 , it is concluded that $H_{\alpha}$ is true and the treatments have a statistically significant effect. Ra obtained from the experiments is compared with the predicted value calculated from the model. Table.2 is an ANOVA summary of the terms in the model, corresponding coefficient of the terms, the standard error of the coefficient, $t$-statistic and $p$-value of the terms to help to decide whether to reject or fail to reject a null hypothesis.

It can be seen that $p$-value of ten terms are below the 0.05 , hence they are significant in the model. The values of $R^{2}$ and $R^{2}$ adj are $100 \%$ and $100 \%$, respectively. Where $R^{2}=100 \%$ indicates that $100 \%$ of total variation in the response that is explained by predictors or factors in the model and $R^{2}$ adj is $100 \%$, which accounts for the number of predictors in the model describes the significance of the relationship. Table. 3 illustrates the ANOVA analysis of the model, and the column describing the degrees of freedom (DF), the sequential sums of squares (Seq. SS), the adjusted sums of squares (Adj SS), the adjusted mean squares (Adj MS), the $F$-statistics from the adjusted mean squares, and its $p$-value. The sequential sum of squares is the added sums of squares given that prior terms are in the model, which depends on the model order.

Table.3 Variance analysis for the fitted model of surface roughness

\begin{tabular}{|c|c|c|c|c|c|c|}
\hline Source & DF & Seq SS & Adj SS & Adj MS & F & P \\
\hline Regression & 14 & 32.0539 & 32.0539 & 2.28957 & 14432.29 & 0.000 \\
\hline Linear & 4 & 31.9035 & 26.7095 & 6.67737 & 42090.81 & 0.000 \\
\hline Square & 4 & 0.0299 & 0.0303 & 0.00757 & 47.69 & 0.000 \\
\hline Interaction & 6 & 0.1206 & 0.1206 & 0.02009 & 126.66 & 0.000 \\
\hline Residual Error & 10 & 0.0016 & 0.0016 & 0.0016 & & \\
\hline Total & 24 & 32.0555 & & & & \\
\hline
\end{tabular}

The adjusted sums of squares are the sums of squares given that all other terms are in the model that does not depend upon the model order. It can be observed in this table, the $p$-value of regression model is less than 0.05 , hence, the Ra fitting the regression model with the linear, and square terms are significant at the level of $95 \%$. The terms Ton $^{2}, V^{2},($ I $p)$ (Toff), (Ton) $($ Toff) and (Toff) $(V)$ are not included in the 0.05 . This way the simplified truncated model is shown in the following equation.

$$
\begin{aligned}
R a= & 5.4035+0.1997(\text { Ip })+0.0268(\text { Ton })+0.0002(\text { Toff })-0.0853(V)+0.0028(I p)^{2} \\
& -0.0005(V)^{2}+0.0006(\text { Ip })(\text { Ton })-0.0017(\text { Ip })(V)-0.0002(\text { Ton })(V)
\end{aligned}
$$

Where $\mathrm{Ra}$ is in $\mu \mathrm{m}$, Ip in Amps and Ton is in $\mu$ s.

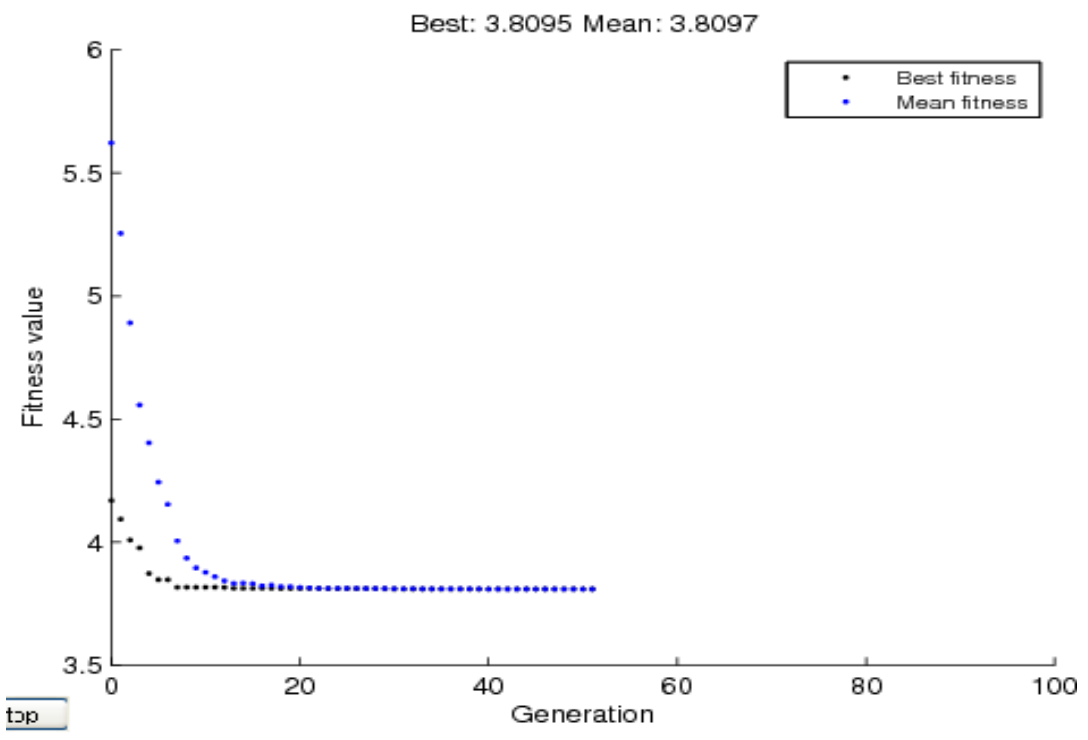

Figure1. Plot of fitness value with number of generations. 
The optimization is carried out in GA of MATLAB environment. The GA parameters were varied to obtain the different optimized process parameters and the optimized GA parameters used for parametric optimization are as follows:

Population type: Double vector; Population size: 100;

Number of generations: 200; Number of stall generation: 50; Fitness function: Rank scaling; Selection function: Roulette wheel, Crossover function: Two point; Crossover fraction: 0.8; Mutation function: adaptive feasible; Migration: Forward, Migration fraction: 0.2. The plot of fitness value with the number of generations is presented in Figure.1.

The best (optimum) material removal condition leading to the minimum surface roughness is shown in Table.4 an experiment was carried out at the optimal parameter settings for surface roughness so that the targeted value of response parameter can be obtained. Table.4 shows the predicted value of surface roughness obtained from the GA and experimental result with the parametric optimal setting as obtained from the GA. Predictions are in good agreement with the experimental results because the percentage error of the predicted value with respect to the experimentally observed values for surface roughness is not high.

Table 4. The optimum value of the process parameters

\begin{tabular}{|c|c|c|c|c|c|c|c|}
\hline \multirow{2}{*}{ Response } & \multicolumn{4}{|c|}{ Optimized value of input parameters } & \multirow{2}{*}{ Predicted Value } & \multirow{2}{*}{ Exp value } & \multirow{2}{*}{$\%$ Error } \\
\hline & Ip & Ton & Toff & $\mathrm{V}$ & & & \\
\hline Surface Roughness (Ra) & 5 & 50 & 575 & 50 & 3.809 & 4.056 & 3.11 \\
\hline
\end{tabular}

\section{Conclusion}

In this article a hybrid GA based RSM techniques have been used to optimize the process parameters of EDM process. A RSM model was applied to develop the mapping between surface roughness and input process parameters. The developed regression model was further coupled with a developed GA to find out the optimum conditions leading to the minimum surface roughness value. The GA has been suitable in optimisation of the response variable. The RSM based surface roughness model can be optimized using GA to obtain the optimum values of independent variables. The predicted optimum material removal condition was validated with an experimental measurement. This result validates the prediction accuracy of GA, because the maximum percentage absolute error of the predicted value with respect to the experimentally observed values for surface roughness is not high. The obtained model can be used to select the level of EDM parameters, and hence a remarkable saving of cost and time.

\section{REFERENCES}

[1] R. Snoeys and F. Van Dyck, "Investigations of EDM operations by means of thermo-mathematical models," CIRP, pp. 33-34., 1971.

[2] K. P. Rajurkar and S. M. Pandit, "Quantitative expressions for some aspects of surface integrity of electro discharge machined components" Journal of Engineering for Industry, vol. 106, No. 2, pp. 171-177, 1984.

[3] B. Bhattacharyya, S. Gangopadhyay, \& B. R. Sarkar, "Modelling and analysis of EDMed job surface integrity”Journal of Materials Processing Technology, vol. 189, pp. 169-177, 2007.

[4] H. Zarepour, A. F. Tehrani, D. Karimi, and S. Amini,
"Statistical analysis on electrode wear in EDM of tool steel DIN 1.2714 used in forging dies," Journal of Materials Processing Technology, vol. 187188, No. 0, pp. $711-714$, 2007.

[5] J. Y. Kao, C. C. Tsao, S. S. Wang, and C. Y. Hsu, "Optimization of the EDM parameters on machining Ti-6Al-4V with multiple quality characteristics," International Journal of Advanced Manufacturing Technology, vol. 47, No. 1-4, pp. 395-402, 2010.

[6] J. A. Sanchez, B. Izquierdo, N. Ortega, I. Pombo, S. Plaza, and I. Cabanes, "Computer simulation of performance of electrical discharge machining operations," International Journal of Computer Integrated Manufacturing, vol. 22, No. 8, pp. 799-811, 2009.

[7] Y. Chen and S. Mahdavian, "Parametric study into erosion wear in a computer numerical controlled electro-discharge machining process," Wear, vol. 236, No. 1, pp. 350-354, 1999.

[8] D. Mandal, S. K. Pal, and P. Saha, "Modeling of electrical discharge machining process using back propagation neural network and multi-objective optimization using non-dominating sorting genetic algorithm- II," Journal of Materials Processing Technology, vol. 186, pp. 154-162, 2007.

[9] M. K. Pradhan, R. Das, and C. K. Biswas, "Prediction of surface roughness in electrical discharge machining of D2 steel using regression and artificial neural networks modeling," Journal of Machining and Forming Technologies, vol. 2, No. 1-2, pp. 25-46, 2009.

[10] M. K. Pradhan and C. K. Biswas, "Investigation into the effect of process parameters on surface roughness in EDM of AISI D2 steel by response surface methodology," International Journal of Precision Technology, vol. 2, pp. 64-80, 2011.

[11] A. Majumder, "Parametric optimization of electric discharge machining by ga-based response surface methodology," Journal of Manuf. Sci. Prod., vol. 12, No. 1, pp. 25-30, 2012. 
[12] C. J. Tzeng and R.-Y. Chen, "Optimization of electric discharge machining process using the response surface methodology and genetic algorithm approach," International Journal of Precision Manufacturing, vol. 14, No. 5, pp.
709-717, 2013.

[13] D. C. Montgomery, Design and analysis of experiments, 4edn. Wiley, New York (1997) 